\title{
Supporting Information for Radioplasmonics: plasmonic transducers in the radiofrequency regime for resonant thermo-acoustic imaging in deep tissues.
}

\author{
Ricardo Martín Abraham-Ekeroth+,‡,n , Francesco De Angelis ${ }^{*}+$ \\ † Istituto Italiano di Tecnologia, Via Morego 30, 16163 Genova, Italy \\ ¥ Instituto de Física Arroyo Seco, IFAS (UNCPBA), Pinto 399, 7000 Tandil, Argentina \\ ॠ CIFICEN (UNCPBA-CICPBA-CONICET), Pinto 399, 7000 Tandil, Argentina
}

Table S1 shows parameters calculated for all the RF-metamaterials simulated (see Figure 2 in the manuscript). In this table we compare the frequencies obtained for the resonances in the spectra from both the polarizability $\alpha$, i.e. equation 3 in the paper, and the absorption cross section $\sigma_{a b s}$ (maxima of the curves) with the purpose to evaluate the influence of the imaginary parts of the permittivities on the spectral positions.

\begin{tabular}{|l|l|l|l|l|}
\hline RF-metamaterial & $\begin{array}{l}\text { Particle } \\
\text { size }(\mu \mathrm{m})\end{array}$ & Frequency $\left(\operatorname{Re}\{\varepsilon\}+2 \varepsilon_{h}=0, \mathrm{MHz}\right)$ & Frequency $\left(\sigma_{a b s,}, \mathrm{MHz}\right)$ & $\begin{array}{l}\varepsilon \\
\text { adimensional })\end{array}$ \\
\hline $\mathrm{CNF} /$ silicone 6 \%wt. & 20 & 7.95 & 9.3 & $-102.7+4.8 \mathrm{i}$ \\
\hline Graphene 3 \% wt./ PDMS & 10 & 19.9 & 20 & $-147.5+1.6 \mathrm{i}$ \\
\hline $\mathrm{APu} 86 \%$ vol. / Al & 10 & 83.11 & 111 & $-123.1+7.2 \mathrm{i}$ \\
\hline $\mathrm{MWCNT} 12 \%$ wt./ $\mathrm{Al}_{2} \mathrm{O}_{3}$ & 10 & 435.7 & 448.5 & $-123.9+105.3 \mathrm{i}$ \\
\hline $\mathrm{Fe}_{78} \mathrm{Si}_{9} \mathrm{~B}_{13} 78 \%$ vol./ epoxy & 10 & 656.6 & 661 & $-146.8+17.1 \mathrm{i}$ \\
\hline $\mathrm{Fe}_{78} \mathrm{Si}_{9} \mathrm{~B}_{13} 83 \%$ vol./ epoxy & 10 & 1046 & 1047 & $-148.4+8.6 \mathrm{i}$ \\
\hline
\end{tabular}

Table S1: Resonant parameters of the radioplasmonic particles given in Figure 2 in the work. The frequencies given in the third column correspond to zeros of the function $\operatorname{Re}\{\varepsilon\}+2 \varepsilon_{h}$ that means truly plasmonic resonances in the polarizability. The frequencies in the fourth column represent the effective plasmonic resonances in the absorption. The last column shows the permittivity of the metamaterials at the frequencies given for the absorption. 
Note that both sets of spectral locations have values roughly close to each other, being the difference due to the nonnegligible imaginary part of the dielectric function in each case. Specifically, the resonances in both expressions mean solutions that are satisfied for complex values. The values for the dielectric function in the particles are given in the last column; they were taken at the frequencies given in the fourth column. We expect that these data could be useful to evaluate

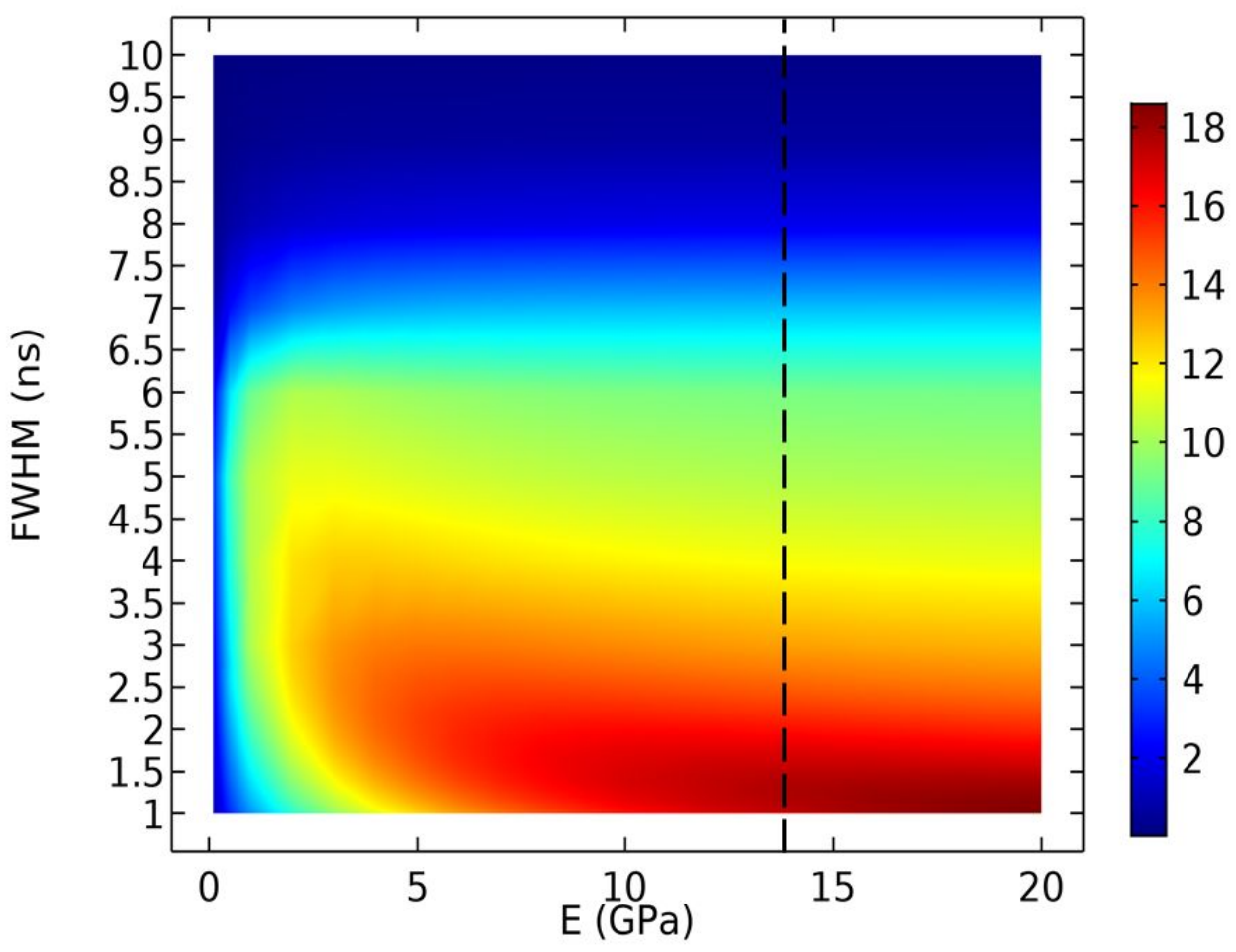

Figure S1: Maximum pressure obtained by photoacoustic effect in MWCNT/ $\mathrm{Al}_{2} \mathrm{O}_{3}$ particles under Gaussian excitation with $130 \mathrm{~mW}$ at a distance $1.1 R$ from the particle center, as a function of FWHM and $E$. Dash line corresponds to the estimation for case $12 \%$ wt..

or compare RF-metamaterials in future experiments. In addition to Figure 5 of the manuscript, we run the parametric variation of the photoacoustic pressure using MWCNT/ $\mathrm{Al}_{2} \mathrm{O}_{3}$ microparticles as a function of the young modulus $E$ and the Full width at half maximum $(F W H M)$ of the incoming pulse. The maximum pressure obtained at a distance $1.1 R$ from the particle center can be seen in Figure S1.

Note that both results from Figure 5 and Figure $\mathrm{S} 1$ follow the same trend of a common physical law. The maximum reached in the scale in Figure $5 \mathrm{a}$ is around 4.5 times the maximum in Figure $\mathrm{S} 1$ for the shortest $F W H M(\sim 1 \mathrm{~ns})$. However, we can see from the results that both RF-metamaterials, i.e. CNF/silicone and MWCNT/ $\mathrm{Al}_{2} \mathrm{O}_{3}$, can be useful for photoacoustic (PA) systems. In this way, we cover a wide range of PA signals with only two types of particles. The responses cover a range of time ranging from a few $n s$ up to $\sim 100 \mathrm{~ns}$ and a set of elastic materials with $E$ ranging from a few $M P a$ up to tens of $G P a$. MWCNT $/ \mathrm{Al}_{2} \mathrm{O}_{3}$ reacts in $\mathrm{GPa}$ while $\mathrm{CNF} /$ silicone reacts in the $\mathrm{MPa}$ range. Both materials complement each other to conceive different designs of $\mathrm{PA}$ applications. 